\title{
Lattice location study of implanted In in Ge
}

\author{
S. Decoster ${ }^{1}$ B. De Vries, ${ }^{1}$ U. Wahl, ${ }^{2}$ J.G. Correia,${ }^{2}$ and A. Vantomme ${ }^{1}$ \\ ${ }^{1}$ Instituut voor Kern- en Stralingsfysica and INPAC, KULeuven, 3001 Leuven, Belgium \\ ${ }^{2}$ UFA, Instituto Tecnológico e Nuclear, Estrada \\ Nacional 10, apt. 21, 2686-953 Sacavém, Portugal
}

(Dated: January 6, 2009)

\begin{abstract}
We report on emission channeling experiments to determine the lattice location and the thermal stability of implanted ${ }^{111} \mathrm{In}$ atoms in Ge. The majority of the In atoms was found on the substitutional site, which is a thermally stable site at least up to $500^{\circ} \mathrm{C}$. We also found strong indication that directly after implantation, a fraction of the implanted ${ }^{111} \mathrm{In}$ atoms occupies the bond-centered site. This fraction disappears after annealing at $300^{\circ} \mathrm{C}$. From comparison with $a b$ initio calculations, electrical studies and perturbed angular correlation experiments, the In atoms on the bond-centered site can be related to In-vacancy and In-self-interstitial defect complexes. The activation energy of this bond-centered related defect was found to be below $1.6 \mathrm{eV}$.
\end{abstract}

PACS numbers: 


\section{INTRODUCTION}

During the past decade, germanium has become an increasingly important material in semiconducting applications. When compared to silicon, Ge has a higher free carrier mobility and a lower dopant activation temperature [1], which makes it an attractive material in metal-oxide semiconductor field-effect transistors $[2,3]$. One of the possible $p$-type dopants in Ge is indium, introducing a shallow acceptor level of $0.0112 \mathrm{eV}$ above the Ge valence band [4]. Since the electrical properties of dopants in semiconductors are drastically influenced by their exact lattice site, a detailed understanding of the lattice site occupation of In atoms in the Ge lattice - together with its thermal stability - is crucial to understand their electrical behavior.

A particular technique that has been applied to investigate the local environment of In atoms in many materials, including Ge, is perturbed angular correlation spectroscopy (PAC). Since the radioactive ${ }^{111} \mathrm{In}$ isotope is the most widely used probe for PAC spectroscopy, it has been frequently used in the past to study the local environment of In atoms in Ge [5-12]. This technique actually probes the surroundings of the daughter atom ${ }^{111} \mathrm{Cd}$, but due to the very small recoil energy $(<1 \mathrm{eV})$ during the radioactive ${ }^{111} \mathrm{In} \rightarrow{ }^{111} \mathrm{Cd}$ decay compared to typical lattice binding energies $(10-20 \mathrm{eV}),{ }^{111} \mathrm{Cd}$ will inherit the lattice location of its precursor ${ }^{111} \mathrm{In}$. After annealing the ${ }^{111} \mathrm{In}$-implanted Ge sample up to $600^{\circ} \mathrm{C}$, no interaction frequency was found in the PAC spectra, showing that all In atoms occupy sites with perfect cubic charge symmetry. Electron irradiation as well as ion irradiation of the annealed ${ }^{111}$ In-doped Ge samples revealed two main defects: one which has been attributed to the In-vacancy (In-V) defect and another one attributed to a defect complex involving an In atom and a Ge self-interstitial $\left(\mathrm{In}-\mathrm{Ge}_{I}\right)$. Both defects have an electric field gradient orientation along the $\langle 111\rangle$-direction and disappear in a temperature range of $110-210^{\circ} \mathrm{C}$. Deep Level Transient Spectroscopy (DLTS) measurements were used in combination with PAC, to investigate the electrical levels in the Ge band gap related to In defects $[12,13]$. This study resulted in a level at $E=E_{V}+0.33$, which was assigned to the In-V defect. As the PAC technique can only give indirect information about the lattice location of the In atoms and as it can not distinguish between In atoms on substitutional (S) or on tetrahedral interstitial (T) sites, both being subject to perfect cubic charge symmetry, other or complementary techniques are required to obtain a complete understanding of the lattice location of the In atoms. In early 
Rutherford channeling spectroscopy (RBS/C) experiments, the majority of the In atoms were found on the S site after annealing $[14,15]$, in accordance with the PAC results where no interaction frequency was found. In order to understand the structural configuration of simple defects such as the In-vacancy defect, ab initio density functional calculations were performed, resulting in In atoms occupying the bond-centered (BC) site [16]. The occupancy of the bond-centered site was calculated for many high-Z impurity atoms in the impurity-vacancy complexes, as well as for $\mathrm{Cd}$ in the $\mathrm{Cd}$-self-interstitial defect complex $[16,17]$. Similar theoretical results have been found for the In-V defect in Si, where the In atom was found to occupy the BC site as well $[16,18]$. Since most of these results on the lattice location of the In atoms in Ge have been obtained through indirect techniques (with the exception of high dose RBS/C experiments) or from calculations that have not been corroborated by experiments so far, in this article, we present a direct lattice location study of ion implanted In in Ge with the emission channeling (EC) technique. The important advantages of this technique with respect to ion channeling techniques (such as RBS/C) are its significantly higher accuracy due to the use of a 2-dimensional detection technique and the use of very low implantation fluences which allows to study isolated atoms.

\section{EXPERIMENTAL METHOD}

In an EC experiment, charged particles emitted from an implanted radioactive isotope, are guided by the potential of atomic rows and planes while traveling through the crystal. The resulting anisotropic electron emission pattern around low-index crystal directions is characteristic for the lattice site occupied by the emitting atom and is measured with a 2-dimensional energy- and position-sensitive Si detector of $22 \times 22$ pixels. More information about this technique can be found in Ref. 19. The radioactive isotope ${ }^{111} \mathrm{In}$ has been implanted at the ISOLDE facility in CERN. It decays with a half life of 2.81 days to ${ }^{111} \mathrm{Cd}$, emitting K (143 and $218 \mathrm{keV}$ ) and L (167 and $241 \mathrm{keV}$ ) conversion electrons. A fluence of $2.9 \times 10^{12}$ In atoms $/ \mathrm{cm}^{2}$ has been implanted with an energy of $60 \mathrm{keV}$ and a beam current density of $200 \mathrm{pA} / \mathrm{cm}^{2}$ in an undoped $\langle 111\rangle$-Ge sample, held at room temperature. The Ge sample was implanted at a tilted angle $\left(10^{\circ}\right)$ with respect to its surface direction to minimize channeled implantation. To obtain accurate and unambiguous results, emission patterns along the four crystal directions $\langle 111\rangle,\langle 100\rangle,\langle 211\rangle$ and $\langle 110\rangle$ were measured, ana- 
lyzed consistently, and fitted to a set of simulated spectra. The simulations are based on the dynamical theory of electron diffraction and have been performed for several high-symmetry sites such as the $\mathrm{S}, \mathrm{BC}$, tetrahedral interstitial $(\mathrm{T})$, hexagonal $(\mathrm{H})$ and the so called $\mathrm{AB}$, SP, Y and C sites, as well as for small discrete displacements between these sites along the $\langle 111\rangle$-, $\langle 100\rangle$ - and $\langle 110\rangle$-direction. The exact location of these sites within the diamond lattice structure and more information about the simulations can be found in Ref. 20 and Ref. 21 respectively. To monitor the thermal stability of the lattice location of the implanted In atoms, the measurements were performed in the as-implanted state as well as after 10 min isochronal annealing steps in vacuum $\left(<10^{-5}\right.$ mbar $)$ up to $500^{\circ} \mathrm{C}$.

\section{RESULTS}

In Fig. 1 (a)-(d) we show the normalized two-dimensional emission yields along the four measured crystal directions after annealing the ${ }^{111} \mathrm{In}$-implanted Ge sample at $400^{\circ} \mathrm{C}$. The high yield (in blue) in the center of each pattern is a signature for the strong channeling effect of the conversion electrons along all crystal axes. This indicates that a large fraction of the In probe atoms is located on the substitutional site. To quantify the fraction of In atoms on the substitutional site, and to investigate whether any other high-symmetry site(s) is (are) present in the spectra, a quantitative fitting procedure with the simulated patterns was performed.

Up to three fractions on different high-symmetry sites were used to obtain the best fit, allowing displaced sites as well. The fitting procedure unambiguously shows that the majority of the In atoms are located on undisturbed substitutional sites - as already inferred from visual inspection of the spectra, as discussed above. It was found that by adding the

high-symmetry sites T, H, AB, SP, Y or C, it is not possible to fit the experimental patterns in all four directions in a consistent way with a significant fit improvement. However, adding a fraction of In atoms on the bond-centered (BC) site, results in consistent fits with relative improvements in $\chi^{2}$ as presented in Fig. 2 (a). These results show that the fit improvement after adding a BC fraction to the fit, is reduced from roughly $3.5 \%$ to less than $1 \%$ for annealing temperatures exceeding $150^{\circ} \mathrm{C}$. This analysis gives strong indication that the addition of a $\mathrm{BC}$ fraction is only relevant for the measurements after implantation and after annealing at $150^{\circ} \mathrm{C}$. Taking this into account, the resulting fraction of atoms on the $\mathrm{S}$ 
$\left(f_{\mathrm{S}}\right)$ and on the $\mathrm{BC}$ site $\left(f_{\mathrm{BC}}\right)$ as a function of annealing temperature, as obtained from the best fits, are shown in Fig. 2 (b). The best fits to the experimental patterns in Fig. 1 (a)-(d) are shown in Fig. 1 (e)-(h), matching the experimental patterns very well. In this fit, 88\% of the implanted In atoms were found to occupy the $\mathrm{S}$ site.

\section{DISCUSSION}

Apart from the fraction of In atoms on the $\mathrm{BC}$ and on the $\mathrm{S}$ site, Fig. 2 further shows the remaining fraction (as calculated from $100 \%-f_{\mathrm{BC}}-f_{\mathrm{S}}$ ) which will be referred to as the random fraction. This random fraction is a direct consequence of implantation-induced lattice damage. Even for the low fluence used in this study, heavy-ion implantation produces highly damaged regions, especially in materials with small lattice binding energies such as Ge. Firstly, due to the deterioration of the crystal structure, a fraction of the implanted radioactive isotopes will be located in damaged regions with reduced local crystallinity. Secondly, a fraction of the conversion electrons emitted from an undamaged region will pass through damaged crystal regions, enhancing the probability for dechanneling. Both effects will result in an isotropic background to the patterns and, consequently, in the random fraction observed in this study. The large random fraction in the as-implanted measurement is due to the presence of implantation-induced crystal lattice damage, while its drastic decrease after annealing at $150^{\circ} \mathrm{C}$ and $300^{\circ} \mathrm{C}$ is a direct consequence of almost complete recrystallization.

The majority of the In atoms are located on the substitutional site, in agreement with the results from the previously mentioned RBS/C and PAC studies [5-7, 9, 11, 14, 15]. The root mean square displacement of the substitutional In atoms was found to be comparable to the expected thermal vibration amplitude at room temperature of $0.07 \AA$. This indicates that the majority of the In atoms on the $\mathrm{S}$ site are embedded in an unperturbed local environment. Besides the fraction of In atoms on the $\mathrm{S}$ site, we also found a significant fit improvement when including a fraction on the bond-centered (BC) site. The fraction of In atoms on the $\mathrm{BC}$ site, relative to the total fraction of In atoms on high-symmetry sites, was found to be $36 \%$ in the as implanted case and $22 \%$ after annealing at $150^{\circ} \mathrm{C}$. This $\mathrm{BC}$ fraction disappears between the $150^{\circ} \mathrm{C}$ and $300^{\circ} \mathrm{C}$ annealing step. In the previously mentioned PAC studies two defects have been identified as being caused by ion irradiation [5-12], and have 
been attributed to In-V and $\mathrm{In}-\mathrm{Ge}_{I}$ defects. These defects were both found to have an electrical field gradient (EFG) along the $\langle 111\rangle$-direction. Since an In atom on the BC site with an axially symmetric surrounding is subject to an EFG along the $\langle 111\rangle$-direction, we have strong indications that the In atoms on the BC site observed in this work, are related to the $\mathrm{In}-\mathrm{V}$ and/or $\mathrm{In}-\mathrm{Ge}_{I}$ defect. Moreover, the temperature range at which these defects disappear in the PAC studies $\left(110-210^{\circ} \mathrm{C}\right)$ is comparable to what is found here, i. e. the $\mathrm{BC}$ fraction partly disappears at $150^{\circ} \mathrm{C}$ and completely vanishes after annealing at $300^{\circ} \mathrm{C}$. Conversely, we would expect the BC fraction to increase similarly to the $\mathrm{S}$ fraction, if it where governed by the recrystallization of the lattice at this temperature, which is clearly not the case as shown in Fig. 2(b). Furthermore, ab initio calculations have shown that in the $\mathrm{In}-\mathrm{V}$, the $\mathrm{Cd}-\mathrm{V}$ and the $\mathrm{Cd}-\mathrm{Ge}_{I}$ defects, the impurity prefers the $\mathrm{BC}$ site to the $\mathrm{S}$ site $[16,17]$. All of these results strongly point towards the fact that the In atoms observed on the $\mathrm{BC}$ site in our study are part of the In-V and/or In- $\mathrm{Ge}_{I}$ defect complex. By introducing vacancies and/or interstitials, e. g. by electron irradiation, it might be possible to even tune the relative fraction of In atoms on the $\mathrm{BC}$ and the $\mathrm{S}$ site with the defect density. This occupancy of the bond-centered site has been observed for some transition metals and for erbium atoms in Ge as well, possibly indicating a more general trend for (several) impurities in Ge: impurity-vacancy and/or impurity-self-interstitial defect complexes with the impurity atom located on the $\mathrm{BC}$ site $[22,23]$.

The occupancy of the BC site within the In-V complex would have some consequence with respect to the diffusion process of In in Ge. In consistency with experimental studies [24, 25], the In atoms are found to diffuse through the bulk Ge by the mediation of vacancies. The proposed diffusion mechanism is called the ring mechanism [26] in which the impurity atoms will change places with vacancies, but will occupy a substitutional site. Recent electronic structure calculations have been used by Chroneos et al. to calculate the activation enthalpies of vacancy-mediated diffusion for several impurity atoms, including indium [27]. However, the activation enthalpy for In $(2.79 \mathrm{eV})$ obtained in that study [27], is inconsistent with experimental values, where a larger value $(3.61 \mathrm{eV})$ was suggested [25]. Chroneos et al. also calculated the actual migration barrier of a vacancy near an In atom, which was found to be $1.18 \mathrm{eV}[27]$.

It can be assumed that the In-V complexes as observed in our work - with the In atom on the bond-centered site with two nearest neighbor vacancies - will play a major role in this 
diffusion model, and that the microscopic behavior of the In atom in the ring mechanism might need revision. From the fact that the $\mathrm{BC}$ fraction disappears completely below $300^{\circ} \mathrm{C}$ we are able to estimate the activation energy for the break-up of the BC-related complex. The activation energy $E_{a}$ is calculated from

$$
E_{a}=k_{B} T_{n} \ln \left[\frac{\nu_{0} \Delta t}{N} \cdot \frac{1}{\ln \left[f\left(T_{n-1}\right) / f\left(T_{n}\right)\right]}\right],
$$

where $k_{B}$ is the Boltzmann constant, $T_{n}$ and $\Delta t$ the temperature and duration of the $n^{\text {th }}$ annealing step, $\nu_{0}$ the attempt frequency (which has been taken $\nu_{0} \approx 10^{12} s^{-1}$, i. e. of the order of the lattice vibrations), $N$ the amount of times the vacancy will be retrapped before it escapes, and $f_{\left(T_{n-1}\right)}$ and $f_{\left(T_{n}\right)}$ the occupied fraction after the $(n-1)^{t h}$ and the $n^{\text {th }}$ annealing step. More background information on this formula can be found in Ref 28 . When using a one-step model without retrapping $(N=1)$ and $300^{\circ} \mathrm{C}$ as the temperature at which the BC-fraction disappears completely, the activation energy was found to be $1.64 \mathrm{eV}$. Increasing the retrapping rate to $N=10$ only lowers this value slightly to $1.53 \mathrm{eV}$. Due to the uncertainty in the temperature at which the $\mathrm{BC}$ fraction is substantially reduced - most likely somewhere between $150^{\circ} \mathrm{C}$ and $300^{\circ} \mathrm{C}$, but it could be even at lower temperatures, since the annealing of the lattice damage at $150^{\circ} \mathrm{C}$ might compensate the decrease of the $\mathrm{BC}$ fraction - we have calculated the activation energy for $T_{n}=200^{\circ} \mathrm{C}$ and $T_{n}=100^{\circ} \mathrm{C}$ as well, resulting in lower activation temperatures of $1.35 \mathrm{eV}$ and $1.07 \mathrm{eV}$ respectively. In this way we obtain a maximum activation energy of $1.64 \mathrm{eV}$ for the break-up of the BC-related complex. In order to relate this value to the literature values for the activation enthalpy of In $[25,27]$ and the migration barrier of a vacancy near an In atom [27], as presented above, more information about the atomic break-up mechanism of this complex is required.

\section{CONCLUSION}

In conclusion, we have determined the lattice location of ion implanted In atoms in Ge together with its thermal stability, using the emission channeling technique. The majority of the In atoms were found to occupy the substitutional site, with a maximum occupation of $90 \%$ after annealing at $300^{\circ} \mathrm{C}$. This site was found to be thermally stable up to at least $500^{\circ} \mathrm{C}$. We found strong indications that In atoms occupy a second high-symmetry, i. e. the bondcentered site, which is thermally stable up to $150^{\circ} \mathrm{C}$. From comparison with previous PAC 
experiments and theoretical studies, this BC fraction can be attributed to In-vacancy and/or In-self-interstitial defect complexes. Moreover, we have determined an upper limit for the activation energy of the BC-related complex around $1.6 \mathrm{eV}$. This In-V complex, where the In atom occupies the $\mathrm{BC}$ site, will be important in the vacancy-mediated diffusion mechanism for In, and possibly also for other impurities in Ge.

\section{ACKNOWLEDGEMENTS}

This work was supported by FWO Flanders (G.0501.07 and G.0636.08), the K.U.Leuven projects GOA/2009/006 and INPAC EF/2005/005, the IUAP P6/42 programme, the Portuguese Foundation for Science and Technology (POCI-FP-81921-2007) and the ISOLDE collaboration. S. D. acknowledges financial support from FWO, Flanders.

[1] R. Hull and J. C. Bean, Germanium Silicon: Physics and Materials, Semiconductors and Semimetals, Academic, San Diego (1999).

[2] C. C. Yeo, B. J. Cho, F. Gao, S. J. Lee, M. H. Lee, C. Y. Yu, C. W. Liu, L. J. Tang, and T. W. Lee, IEEE Electron Device Letters 26, 761 (2005).

[3] Y. J. Yang, W. S. Ho, C. F. Huang, S. T. Chang, and C. W. Liu, Appl. Phys. Lett. 91, 102103 (2007).

[4] T. H. Geballe and F. J. Morin, Phys. Rev. 95, 1085 (1954).

[5] U. Feuser, R. Vianden, and A. F. Pasquevich, Hyperfine Interactions 60, 829 (1990).

[6] U. Feuser, R. Vianden, E. Alves, M. F. da Silva, E. Szilagyi, F. Paszti, and J. C. Soares, Nucl. Inst. Meth. Phys. Res. B 59, 1049 (1991).

[7] H. Haesslein, R. Sielemann, M. Brüßler, and H. Metzner, Hyperfine Interactions 84, 65 (1994).

[8] H. Haesslein, R. Sielemann, and C. Zistl, Materials Science Forum 258, 59 (1997).

[9] H. Haesslein, R. Sielemann, and C. Zistl, Phys. Rev. Lett. 80, 2626 (1998).

[10] R. Sielemann, H. Haesslein, L. Wende, and C. Zistl, Physica B 273, 565 (1999).

[11] C. J. Glover, A. P. Byrne, and M. C. Ridgway, Nucl. Inst. Meth. Phys. Res. B 175, 51 (2001).

[12] R. Sielemann, H. Hässlein, C. Zistl, M. Müller, L. Stadler, and V. V. Emtsev, Physica B 308, 529 (2001). 
[13] C. Zistl, R. Sielemann, H. Hässlein, S. Gall, D. Bräunig, and J. Bollmann, Materials Science Forum 258, 53 (1997).

[14] K. Björkqvist, B. Domeij, L. Eriksson, G. Fladda, A. Fontell, and J. W. Mayer, Appl. Phys. Lett. 13, 379 (1968).

[15] K. Björkqvist, D. Sigurd, G. Fladda, and G. Bjarnholt, Radiat. Eff. 6, 141 (1970).

[16] H. Höhler, N. Atodiresei, K. Schroeder, R. Zeller, and P. H. Dederichs, Phys. Rev. B 71, $035212(2005)$.

[17] H. Höhler, N. Atodiresei, K. Schroeder, R. Zeller, and P. H. Dederichs, Phys. Rev. B 70, $155313(2004)$.

[18] P. Alippi, A. La Magna, S. Scalese, and V. V. Privitera, Phys. Rev. B 69, 085213 (2004).

[19] U. Wahl, J. G. Correia, A. Czermak, S. G. Jahn, P. Jalocha, J. G. Marques, A. Rudge, F. Schopper, J. C. Soares, A. Vantomme, et al., Nucl. Inst. Meth. Phys. Res. A 524, 245 (2004).

[20] U. Wahl, Phys. Rep. 280, 145 (1997).

[21] H. Hofsäss and G. Lindner, Phys. Rep. 201, 121 (1991).

[22] S. Decoster, B. De Vries, A. Vantomme, U. Wahl, and J. G. Correia, Appl. Phys. Lett. 93, 141907 (2008).

[23] S. Decoster, S. Cottenier, B. De Vries, H. Emmerich, U. Wahl, J. G. Correia, and A. Vantomme, unpublished (2008).

[24] V. A. Pantaleev, Soviet Physics - Solid State 7, 734 (1965).

[25] P. Dorner, W. Gust, A. Lodding, H. Odelius, B. Predel, and U. Roll, Z. Metallkunde 73, 325 (1982).

[26] S. M. Hu, phys. stat. sol. (b) 60, 595 (1973).

[27] A. Chroneos, H. Bracht, R. W. Grimes, and P. Uberuaga, Appl. Phys. Lett. 92, 172103 (2008).

[28] U. Wahl, J. G. Correia, E. Rita, J. P. Araújo, J. C. Soares, and the ISOLDE Collaboration, Nucl. Inst. Meth. Phys. Res. B 253, 167 (2006). 


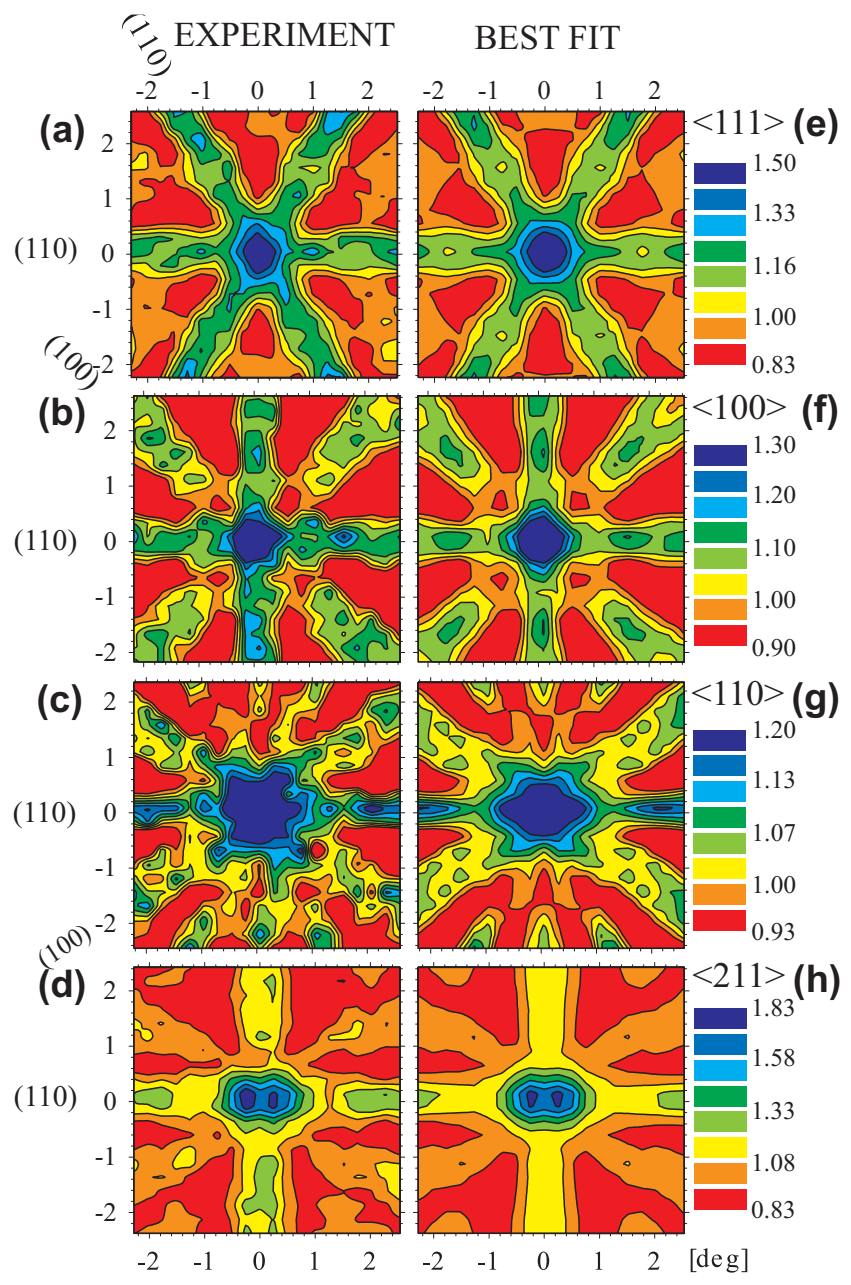

FIG. 1: (Color online)(a)-(d) Two-dimensional normalized conversion electron patterns emitted from ${ }^{111} \mathrm{In}$ in $\mathrm{Ge}$ around the $\langle 111\rangle,\langle 100\rangle,\langle 110\rangle$ and $\langle 211\rangle$-axes, following a $400^{\circ} \mathrm{C}$ annealing in vacuum; (e)-(h) Best fits to the experimental patterns. The anisotropy is depicted with a color scale between red (low) and blue (high). 


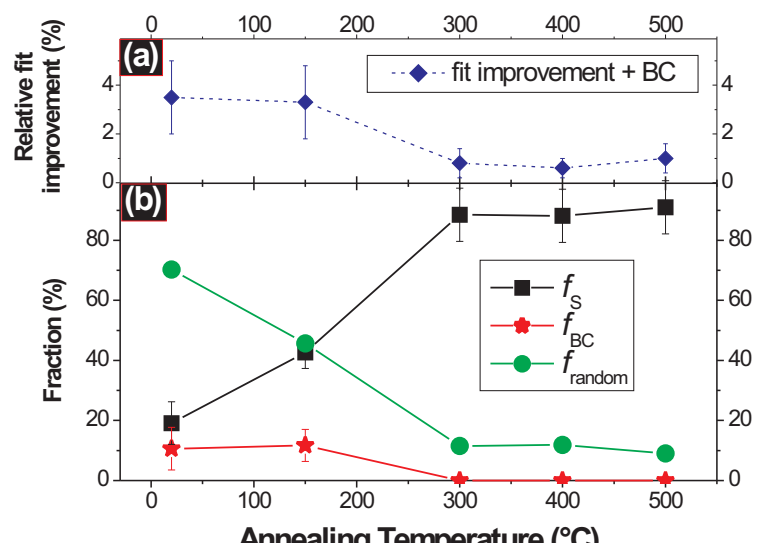

FIG. 2: (Color online) (a) Relative improvement in $\chi^{2}(\%)$ as a function of annealing temperature when adding a $\mathrm{BC}$ fraction to the fit with purely substitutional In atoms; (b) the fraction of In atoms on the $\mathrm{S}$ site $\left(f_{\mathrm{S}}\right.$ - black squares $)$ and on the $\mathrm{BC}$ site $\left(f_{\mathrm{BC}}\right.$ - red stars $)$, together with the random fraction ( $f_{\text {random }}$ - green circles), as a function of annealing temperature. 